ACTA ARITHMETICA

$\mathrm{X}(1965)$

\title{
Über reduzible Folgen
}

von

A. SÁRKöZY (Budapest)

Die Definitionen bzw. Bezeichnungen, die ich in dieser Arbeit benütze, sind in Aufsatz [1] zu finden.

Ich habe in Aufsatz [1] bewiesen, daß - eine beliebige Folge $\mathfrak{U}$ vorgebend - durch Veränderung von $O\left(\frac{n \log \log n}{\log n}\right)$ Elementen der Folge stets erreicht werden kann, daß die veränderte Folge totalprimitiv wird.

P. Erdös wirft die Frage auf, ob folgende Verschärfung des erwähnten Satzes richtig sei: kann man im Falle einer beliebig gegebenen Funktion $f(x)$, für die $\lim f(x)=+\infty$ gilt, and einer beliebig gegebenen Folge durch Veränderung von $O(f(n))$ Elementen der Folge stets erreichen, daß die veränderte Folge totalprimitiv wird?

Ich werde beweisen, daß diese Behauptung nicht stichhaltig ist; und zwar beweise ich, daß es eine derartige Folge (die Folge der nichtnegativen ganzen Zahlen) gibt, die, wie auch immer wir ,,zu wenig", Elemente verändern mögen, reduzibel bleibt.

SATZ I. Ist $e(c>0)$ hinreichend klein, dann ist jede Folge $\mathfrak{C}=$ $=\left\{c_{1}, c_{2}, \ldots\right\}$, für die für $n \geqslant 3$

$$
(n+1)-C(n)<c \sqrt[3]{\frac{n(\log \log n)^{2}}{(\log n)^{4}}}
$$

gilt, reduzibel.

Beweis. Wir liefern einen teilweise konstruktiven Beweis. Die Konstruktion werden wir durch Rekursion durchführen.

Bezeichnen wir die Folge der nichtnegativen ganzen Zahlen mit $\mathfrak{N}: \mathfrak{N}=\{0,1,2, \ldots\}$. Nehmen wir an, die Folgen $\mathfrak{U}^{\left(k_{0}\right)}, \mathfrak{U}^{\left(k_{0}+1\right)}, \ldots, \mathfrak{U}^{(k)}$ bzw. $\mathfrak{Z}^{\left(k_{0}\right)}, \mathfrak{Z}^{\left(k_{0}+1\right)}, \ldots, \mathfrak{Z}^{(k)}$ seien gegeben, und befriedigen folgende Eigenschaften:

1)

(1)

$$
\mathfrak{U}^{(i)} \subset\left(\left[0 ; 2^{i}\right] \cap \mathfrak{M}\right) \quad\left(i=k_{0}, k_{0}+1, \ldots, k\right)
$$


und

2)

(3)

$$
\mathfrak{B}^{(i)} \subset\left(\left[0 ; 2^{i}\right] \frown \mathfrak{N}\right) \quad\left(i=k_{0}, k_{0}+1, \ldots, k\right) .
$$

4)

(5) $\quad B^{(i)}\left(2^{j}\right)=\left[\sqrt{2^{j}}\right] \quad\left(i=k_{0}, k_{0}+1, \ldots, k ; j=0,1,2, \ldots, i\right)$.

5)

$$
\mathfrak{U}^{(i)}+\mathfrak{B}^{(i)} \subset\left(\left[0 ; 2^{i+1}\right] \frown \mathfrak{C}\right) \quad\left(i=k_{0}, k_{0}+1, \ldots, k\right) .
$$

6) $\mathfrak{U}^{(i)} \cap\left[0 ; \frac{1}{48 c} \sqrt[3]{\frac{2^{2 i-2}\left(\log 2^{i-1}\right)^{4}}{\left(\log \log 2^{i-1}\right)^{2}}}\right]$

$$
=\mathfrak{U}^{(i-1)} \curvearrowleft\left[0 ; \frac{1}{48 c} \sqrt[3]{\frac{2^{2 i-2}\left(\log 2^{i-1}\right)^{4}}{\left(\log \log 2^{i-1}\right)^{2}}}\right] \quad\left(i=k_{0}+1, k_{0}+2, \ldots, k\right) .
$$

6)

(7) $\quad \mathfrak{B}^{(i)} \cap\left[0 ; 2^{i-1}\right]=\mathfrak{B}^{(i-1)} \quad\left(i=k_{0}+1, k_{0}+2, \ldots, k\right)$.

7) Ist $a \leqslant 2^{i}$ and $a \notin \mathfrak{U}^{(i)}$, dann existiert ein $b_{j}^{(i)} \epsilon \mathfrak{B}^{(i)}$, so daß $b_{j}^{(i)}+a \notin \Subset$ ist $\left(i=k_{0}, k_{0}+1, \ldots, k\right)$.

8) Für alle $0<d \leqslant 2^{i}$ ist die Anzahl der verschiedenen Lösungen

(8)

$$
b_{j}^{(i)}-b_{l}^{(i)}=d
$$

kleiner, als

$$
\varphi(i) \stackrel{\text { def }}{=} \frac{1}{32 c^{3 / 2}} \cdot \frac{\left(\log 2^{i}\right)^{2}}{\log \log 2^{i}} \quad\left(i=k_{0}, k_{0}+1, \ldots, k\right) .
$$

Vor allem, wenn $\theta$ genügend klein ist, so existieren offensichtlich solche Folgen $\mathfrak{U}^{\left(k_{0}\right)}$ und $\mathfrak{Z}^{\left(k_{0}\right)}$, die von diesen acht Eigenschaften jene befriedigen, die auch bei $k=k_{0}$ Sinn haben, d. $h$. die Eigenschaften 1), 2), 3), 4), 7) und 8).

Es sei nämlich für ein beliebiges $k_{0} \geqslant 2, \mathfrak{U}^{\left(k_{0}\right)}=\left[0 ; 2^{k_{0}}\right] \cap \mathfrak{N}$, und es sei $\mathfrak{B}^{\left(k_{0}\right)}$ durch $0 \in \mathfrak{Z}^{\left(k_{0}\right)}$ und $B^{\left(k_{0}\right)}(i)=[\sqrt{i}]\left(i=1,2, \ldots, 2^{k_{0}}\right)$ definiert. Wenn $c$ genügend klein ist, es gilt offensichtlich

und

$$
\left[0 ; 2^{k_{0}+1}\right] \frown \mathcal{C}=\left[0 ; 2^{k_{0}+1}\right] \frown \mathfrak{M}
$$

$$
\varphi\left(k_{0}\right)>k_{0} .
$$

Wählen wir $c$ so, daß (9) und (10) erfüllt werden, dann ist leicht zu sehen, daß $\mathfrak{U}^{\left(k_{0}\right)}$ und $\mathfrak{B}^{\left(k_{0}\right)}$ die erwähnten Eigenschaften befriedigen.
Offensichtlich folgt aus der Konstruktion, daß man, eine beliebig große natürliche Zahl $N$ vorgebend, diese Zahl auf die erwähnte Weise immer als $k_{0}$ wählen kann, wenn $c$ genügend klein ist (wir werden uns auf diese Bemerkung noch öfter berufen).

Wir müssen noch beweisen, daß man, wenn $\mathfrak{U}^{\left(k_{0}\right)}, \mathfrak{U}^{\left(k_{0}+1\right)}, \ldots, \mathfrak{U}^{(k)}$ und $\mathfrak{Z}^{\left(k_{0}\right)}, \mathfrak{Z}^{\left(k_{0}+1\right)}, \ldots, \mathfrak{Z}^{(k)}$ schon definierte sind, $\mathfrak{U}^{(k+1)}$ und $\mathfrak{Y}^{(k+1)}$ so definieren kann, daß sie die obigen acht Eigenschaften befriedigen.

Bezeichnen wir mit $H^{(k)}$ die Menge der Zahlen $n$, für die

$$
2^{k}<n \leqslant 2^{k+1}
$$

erfüllt ist, und für die für $0 \leqslant i \leqslant f(k)$

ist, wo

$$
n+i \in \mathbb{C} \quad(i=0,1,2, \ldots,[f(k)])
$$

$$
f(k) \stackrel{\text { def }}{=} \frac{1}{12 c} \sqrt[3]{\frac{2^{2 k}\left(\log 2^{k}\right)^{4}}{\left(\log \log 2^{k}\right)^{2}}}
$$

ist.

Bezeichnen wir die Anzahl der Elemente von $H^{(k)}$ mit $H(k)$. Wir werden beweisen, da $\beta$ - wenn $k_{0}$ hinreichend gro $\beta$ war (wie wir erwähnten, kann man das mit hinreichend kleinem $c$ erreichen) - für jedes $k \geqslant k_{0}$

$$
H(k)>2^{k-1}
$$

erfüllt ist.

Ist nämlich für irgendein $n(11)$ und mit geeignetem $0 \leqslant i \leqslant f(k)$, $n+i_{\notin} \mathfrak{C}$ erfüllt, so ist offensichtlich

$$
n \epsilon \bigcup_{j=1}^{\bar{C}\left(2^{k+2}+f(k)\right)}\left[\bar{c}_{j}-f(k) ; \bar{c}_{j}\right]
$$

(wobei $\overline{\mathfrak{S}}=\left\{\bar{c}_{1}, \bar{c}_{2}, \ldots\right\}$ das auf die Folge $\mathfrak{N}$ bezügliche Komplement der Folge $\left(\mathcal{C}\right.$ ist). Hier steht die Vereinigung solcher $\bar{C}\left(2^{k+2}+f(k)\right)$ Intervalle, die je $[f(k)+1]$ ganze Zahlen enthalten, also enthält ihre Vereinigung höchstens $\bar{C}\left(2^{k+2}+f(k)\right)(f(k)+1)$ ganze Zahlen; somit ist die Anzahl der ganzen Zahlen $n$, für die (11) und mit irgendeinem $0 \leqslant i$ $\leqslant f(k) n+i \notin \mathbb{E}$ erfüllt ist, höchstens $\bar{C}\left(2^{k+2}+f(k)\right)(f(k)+1)$.

So ergibt sich für die Anzahl der (11) und (12) befriedigenden Zahlen die folgende Unterschätzung:

$$
\begin{aligned}
H(k) & >\left(2^{k+1}-2^{k}\right)-\bar{C}\left(2^{k+2}+f(k)\right)(f(k)+1) \\
& =2^{k}-\bar{C}\left(2^{k+2}+f(k)\right)(f(k)+1) .
\end{aligned}
$$


Ist $k_{0}$ und somit $k$ hinreichend groß, so ist $f(k)>1$ und

$$
\begin{aligned}
\bar{C}\left(2^{k+2}+f(k)\right) & <c \sqrt[3]{\frac{\left(2^{k+2}+f(k)\right)\left(\log \log \left(2^{k+2}+f(k)\right)\right)^{2}}{\left(\log \left(2^{k+2}+f(k)\right)\right)^{4}}} \\
& <c \sqrt[3]{4} \sqrt[3]{1+\frac{f(k)}{2^{k+2}}} \sqrt[3]{\frac{2^{k}\left(\log \log 2^{k}\right)^{2}}{\left(\log 2^{k}\right)^{4}}} \\
& =c \sqrt[3]{4} \sqrt[3]{1+o(1)} \sqrt[3]{\frac{2^{k}\left(\log \log 2^{k}\right)^{2}}{\left(\log 2^{k}\right)^{4}}}<3 e \sqrt[3]{\frac{2^{k}\left(\log \log 2^{k}\right)^{2}}{\left(\log 2^{k}\right)^{4}}}
\end{aligned}
$$

hiermit folgt aus (14)

$$
\begin{aligned}
H(k) & >2^{k}-\bar{C}\left(2^{k+2}+f(k)\right)(f(k)+1)>2^{k}-\bar{C}\left(2^{k+2}+f(k)\right) 2 f(k) \\
& >2^{k}-3 c \sqrt[3]{\frac{2^{k}\left(\log \log 2^{k}\right)^{2}}{\left(\log 2^{k}\right)^{4}}} \cdot 2 \cdot \frac{1}{12 c} \sqrt[3]{\frac{2^{2 k}\left(\log 2^{k}\right)^{4}}{\left(\log \log 2^{k}\right)^{2}}} \\
& =2^{k}-\frac{1}{2} \sqrt[3]{2^{k}}=2^{k}-2^{k-1}=2^{k-1}
\end{aligned}
$$

und damit ist (13) bewiesen.

Bezeichnen wir jene Teilmenge von $H^{(k)}$, die genau

$$
q(k) \stackrel{\text { def }}{=}\left[\sqrt{2^{k+1}}\right]-\left[\sqrt{2^{k}}\right]
$$

Elemente enthalten, mit $\left.G^{(k, 1)}\right), G^{(k, 2)}, \ldots, G^{\left(k, N_{k}\right)}$, und bezeichnen wir jene Menge, deren Elemente $G^{(k, 1)}, G^{(k, 2)}, \ldots, G^{\left(k, N_{k}\right)}$ sind, mit $\Gamma^{(k)}$ : $\Gamma^{(k)}=\left\{G^{(k, i)}\right\}_{i=1}^{N_{k}}$. Es sei $G^{(k, i)}=\left\{g_{1}^{(i)}, g_{2}^{(i)}, \ldots, g_{q(k)}^{(i)}\right\}$.

Wir werden beweisen, daß $\Gamma^{(k)}$ ein solches Element $G^{(k, i)}$ enthält, so daß die Summe der Lösungzahlen der Gleichungen

und

$$
\begin{gathered}
g_{x}^{(k, i)}-g_{y}^{(k, i)}=d, \\
g_{z}^{(k, i)}-b_{t}^{(k)}=d \\
b_{r}^{(k)}-b_{s}^{(k)}=d
\end{gathered}
$$

für jedes $0<d \leqslant 2^{k+1}$ kleiner ist, als $\varphi(k+1)$.

Um diese Behauptung zu beweisen, werden wir für die Anzahl der ,schlechten" Mengen $G^{(k, i)}$, d. h. für die Anzahl der Mengen $G^{(k, i)}$, für die für irgendein $d$ die Gleichungen (15), (16) und (17) insgesamt mindestens $\varphi(k+1)$ Lösungen haben, eine Überschätzung geben.

Da die Lösungzahl von (17) auf Grund der Voraussetzung der Induktion der achten Eigenschaft nach kleiner ist, als $\varphi(k)$, für eine ,sshlech- te" Menge $G^{(k, i)}$ ist die Lösungzahl von (15) oder (16) bei geeignetem $d$ größer, als

$$
\left[\frac{\varphi(k+1)-\varphi(k)}{2}\right] \stackrel{\text { def }}{=} \delta(k) \text {. }
$$

Bei fixem $d$ aber können in (16) $\delta(k) b_{t}^{(k)}$ auf höchstens $\left(\begin{array}{c}B^{(k)}\left(2^{k}\right) \\ \delta(k)\end{array}\right)$-erlei Weise gewählt werden, und zu diesen ist $g_{z}^{(k, i)}$ schon eindeutig definiert; weitere $q(k)-\delta(k)$ Elemente einer, ,schlechten" Menge $G^{(k, i)}$ können aus $H^{(k)}$ auf höchstens $\left(\begin{array}{c}H(k) \\ q(k)-\delta(k)\end{array}\right)$-erlei Weise gewählt werden; hiermit ist bei fixem $a$ die Anzahl der Mengen $G^{(k, i)}$, für die (16) mehr, als $\delta(k)$ Lösungen hat, höchstens

$$
\left(\begin{array}{c}
B^{(k)}\left(2^{k}\right) \\
\delta(k)
\end{array}\right) \cdot\left(\begin{array}{c}
H(k) \\
q(k)-\delta(k)
\end{array}\right) .
$$

Da $d$ höchstens $2^{k+1}$ verschiedene Werte annehmen kann, ist die Anzahl der Mengen $G^{(k, i)}$, für die (16) bei irgendeinem $d$ mehr, als $\delta(k)$ Lösungen
hat, höchstens

$$
2^{k+1}\left(\begin{array}{c}
B^{(k)}\left(2^{k}\right) \\
\delta(k)
\end{array}\right)\left(\begin{array}{c}
H(k) \\
q(k)-\delta(k)
\end{array}\right) .
$$

Ähnlich können bei fixem $d$ in (15) $\delta(k) g_{z}^{(k, i)}$ auf höchstens $\left(\begin{array}{c}H(k) \\ \delta(k)\end{array}\right)$-erlei Weise gewählt werden, und zu diesen ist $g_{x}^{(k, i)}$ nunmehr eindeutig definiert; weitere $q(k)-2 \delta(k)$ Elemente einer ,schlechten" Menge $G^{(k, i)}$ können auf $\left(\begin{array}{c}H(k) \\ q(k)-2 \delta(k)\end{array}\right)$-erlei Weise gewählt werden. Somit ist bei fixem $d$ die Anzahl der Mengen $G^{(k, i)}$, für die (15) mehr, als $\delta(k)$ Lösungen hat,
höchstens

$$
\left(\begin{array}{l}
H(k) \\
\delta(k)
\end{array}\right)\left(\begin{array}{c}
H(k) \\
q(k)-2 \delta(k)
\end{array}\right)
$$

die Anzahl all dieser Mengen also höchstens

$$
2^{k}\left(\begin{array}{l}
H(k) \\
\delta\left(l_{c}\right)
\end{array}\right)\left(\begin{array}{c}
H(k) \\
q(k)-2 \delta(k)
\end{array}\right) .
$$

So ist die Anzahl sämtlicher, ,schlechter" Mengen $G^{(k, i)}$ höchstens

$$
2^{k+1}\left(\begin{array}{c}
B^{(k)}\left(2^{k}\right) \\
\delta(k)
\end{array}\right)\left(\begin{array}{c}
H(k) \\
q(k)-\delta(k)
\end{array}\right)+2^{k}\left(\begin{array}{l}
H(k) \\
\delta(k)
\end{array}\right)\left(\begin{array}{c}
H(k) \\
q(k)-2 \delta(k)
\end{array}\right) \stackrel{\text { def }}{=} \varrho(k) .
$$

Es ist offensichtlich $N_{k}=\left(\begin{array}{l}H(k) \\ q(k)\end{array}\right)$; wir werden beweisen, daß - wenn $k_{0}$ hinreichend groß ist - für jedes $k \geqslant k_{0}$

$$
\varrho(k)<\left(\begin{array}{l}
H(k) \\
q(k)
\end{array}\right) \quad\left(=N_{k}\right) .
$$


Ist $k_{0}$ und so $k$ hinreichend groß, so gilt offensichtlich

(19) $\delta(k)=\left[\frac{\varphi(k+1)-\varphi(k)}{2}\right]$

$$
\begin{aligned}
& =\left[\frac{1}{64 c^{3 / 2}}\left(\frac{\left(\log 2^{k+1}\right)^{2}}{\log \log 2^{k+1}}-\frac{\left(\log 2^{k}\right)^{2}}{\log \log 2^{k}}\right)\right] \\
& =\left[\frac { 1 } { 6 4 c ^ { 3 / 2 } } \left(\frac{\left(\log 2^{k}\right)^{2}}{\log \log 2^{k+1}}-\frac{\left(\log 2^{k}\right)^{2}}{\log \log 2^{k}}+\right.\right. \\
& \left.\left.\quad+\frac{2 \log 2^{k} \log 2}{\log \log 2^{k+1}}+\frac{(\log 2)^{2}}{\log \log 2^{k+1}}\right)\right] \\
& =\left[\frac{1}{64 c^{3 / 2}}\left(O\left(\frac{\log 2^{k}}{\left(\log \log 2^{k}\right)^{2}}\right)+\frac{\log 4 \log 2^{k}}{\log \log 2^{k+1}}+o(1)\right)\right] \\
& =\left[\frac{1}{64 c^{3 / 2}} \cdot \frac{\log 4 \log 2^{k}}{\log \log 2^{k}}(1+o(1))\right]>\frac{1}{64 c^{3 / 2}} \cdot \frac{\log 2^{k}}{\log \log 2^{k}} .
\end{aligned}
$$

So - wenn $k_{0}$ hinreichend groß ist - gilt auf Grund von (5), (13) und

$$
q(k) \sim \sqrt{2^{k+1}}-\sqrt{2^{k}}<2^{k-2}<\frac{1}{2} H(k)
$$

$$
\begin{aligned}
\varrho(k) & <2^{k+1}\left(\begin{array}{l}
H(k) \\
q(k)
\end{array}\right) \times \\
& \times\left(\left(\begin{array}{c}
B^{(k)}\left(2^{k}\right) \\
\delta(k)
\end{array}\right) \frac{q(k)(q(k)-1) \ldots(q(k)-\delta(k)+1)}{(H(k)-q(k)+1)(H(k)-q(k)+2) \ldots(H(k)-q(k)+\delta(k))}+\right. \\
& \left.+\left(\begin{array}{c}
H(k) \\
\delta(k)
\end{array}\right) \frac{q(k)(q(k)-1) \ldots(q(k)-2 \delta(k)+1)}{(H(k)-q(k)+1)(H(k)-q(k)+2) \ldots(H(k)-q(k)+2 \delta(k))}\right) \\
< & 2^{k+1}\left(\begin{array}{l}
H(k) \\
q(k)
\end{array}\right)\left(\frac{1}{(\delta(k)) !}\left(\frac{B^{(k)}\left(2^{k}\right) q(k)}{H(k)-q(k)}\right)^{\delta(k)}+\frac{1}{(\delta(k)) !}\left(\frac{H(k) q^{2}(k)}{(H(k)-q(k))^{2}}\right)^{\delta(k)}\right) \\
< & \frac{2^{k+1}}{(\delta(k)) !}\left(\begin{array}{l}
H(k) \\
q(k)
\end{array}\right)\left(\left(\frac{2 B^{(k)}\left(2^{k}\right) q(k)}{H(k)}\right)^{\delta(k)}+\left(\frac{4 H(k) q^{2}(k)}{H^{2}(k)}\right)^{\delta(k)}\right) \\
= & \frac{2^{k+1}}{(\delta(k)) !}\left(\begin{array}{l}
H(k) \\
q(k)
\end{array}\right)\left(\left(\frac{2 B^{(k)}\left(2^{k}\right) q(k)}{H(k)}\right)^{\delta(k)}+\left(\frac{4 q^{2}(k)}{H(k)}\right)^{\delta(k)}\right) \\
< & \frac{2^{k+1}}{(\delta(k)) !}\left(\begin{array}{l}
H(k) \\
q(k)
\end{array}\right)\left(\left(\frac{2 \sqrt{2^{k}} \sqrt{2^{k+1}}}{2^{k-1}}\right)^{\delta(k)}+\left(\frac{4\left(\sqrt{2^{k+1}}\right)^{2}}{2^{k-1}}\right)^{\delta(k)}\right) \\
= & \frac{2^{k+1}}{(\delta(k)) !}\left(\begin{array}{l}
H(k) \\
q(k)
\end{array}\right)\left((4 \sqrt{2})^{\delta(k)}+16^{\delta(k)}\right)<\frac{2 \cdot 2^{k+1}}{(\delta(k)) !} 16^{\delta(k)}\left(\begin{array}{l}
H(k) \\
q(k)
\end{array}\right) .
\end{aligned}
$$

Aus der Stirlingschen Formel folgt, daß für hinreichend großes $k_{0}$

$$
(\delta(k)) !>(\delta(k))^{\delta(k)} / 3^{\delta(k)}
$$

ist, also auf Grund von (19) für hinreichend großes $k_{0}$

$$
\begin{gathered}
\varrho(k)<\frac{2^{k+2} 48^{\delta(k)}}{(\delta(k))^{\delta(k)}}\left(\begin{array}{l}
H_{(k)}^{\prime}(k) \\
q(k)
\end{array}\right)=\left(\begin{array}{l}
H(k) \\
q(k)
\end{array}\right) \exp \left((k+2) \log 2-\delta(k) \log \frac{\delta(k)}{48}\right) \\
<\left(\begin{array}{l}
H(k) \\
q(k)
\end{array}\right) \exp \left((k+2) \log 2-\frac{1}{64 e^{3 / 2}} \cdot \frac{\log 2^{k}}{\log \log 2^{k}} \cdot \log \frac{\log 2^{k}}{48 \cdot 64 e^{3 / 2} \log \log 2^{k}}\right) \\
=\left(\begin{array}{l}
B(k) \\
q(k)
\end{array}\right) \exp \left(k \log 2-\frac{k \log 2}{64 e^{3 / 2}}\left(1-\frac{\log \log \log 2^{k}+\log 48 \cdot 64 e^{3 / 2}}{\log \log 2^{k}}\right)+2 \log 2\right) .
\end{gathered}
$$

Hier ist der Exponent von $e$ beliebig klein (gleichmäßig für alle $k \geqslant k_{0}$ ), wenn $c$ hinreichend klein ist, für hinreichend kleines $c$ also kleiner, als 0, und in diesem Falle gilt (18). Damit ist (18) bewiesen.

Die linke Seite von (18) ist nicht kleiner, als die Anzahl der Elemente von $\Gamma^{(k)}$, für die für mindestens ein $d$ die Summe der Lösungzahlen von (15), (16) und (17) mindestens $\varphi(k+1)$ ist, also existiert ein $G^{\left(k, i_{0}\right)} \in \Gamma^{(k)}$, für das die Summe der Lösungzahlen von (15), (16) und (17) kleiner ist, als $\varphi(k+1)$.

$$
\text { Es sei }
$$

ferner

$$
\mathfrak{Z}^{(k+1)} \stackrel{\text { def }}{=} \mathfrak{B}^{(k)} \cup G^{\left(k, i_{0}\right)},
$$

schließlich sei $\mathfrak{U}^{(k+1)} \frown\left(\frac{1}{4} f(k) ; 2^{k+1}\right]$ so definiert, daß $n \in \mathfrak{U}^{(k+1)} \quad\left(\frac{1}{4} f(k)<n\right.$ $\left.\leqslant 2^{k+1}\right)$ dann und nur dann erfüllt ist, wenn für jedes $1 \leqslant i \leqslant B^{(k+1)}\left(2^{k+1}\right)$, $n+b_{i}^{(k+1)} \epsilon \mathbb{C}$ erfüllt ist.

Ich werde beweisen, daß die Folgen $\mathfrak{2}^{(k+1)}$ und $\mathfrak{Z}^{(k+1)}$ die acht erforderten Eigenschaften besitzen.

Da nunmehr diese acht Eigenschaften für $k_{0} \leqslant i \leqslant k$ der Voraussetzung der Induktion nach exfüllt sind, genügt es, sich auf $i=k+1$ zu beschränken.

Die Erfüllung von (1) und (2) ist trivial.

Aus $(20)$ und $G^{\left(k, i_{0}\right)} \subset H^{(k)} \subset\left(2^{k} ; 2^{k+1}\right]$ folgt, daß

$$
\mathfrak{Z}^{(k+1)} \cap\left[0 ; 2^{k}\right]=\mathfrak{B}^{(k)}
$$

ist, und so ist (5) für $0 \leqslant j \leqslant k$ der Voraussetzung der Induktion gemäß erfüllt; ist jedoch $j=k+1$, so folgt wegen $\mathfrak{B}^{(k)} \cap G^{\left(k, i_{0}\right)}=0$ aus (20)

$$
B^{(k+1)}\left(2^{k+1}\right)=B^{(k)}\left(2^{k}\right)+q(k)=\left[\sqrt{2^{k}}\right]+\left(\left[\sqrt{2^{k+1}}\right]-\left[\sqrt{2^{k}}\right]\right)=\left[\sqrt{2^{k+1}}\right],
$$

(5) ist also auch jetzt erfüllt. 
(6) ist nach (21) erfüllt. trivial.

Die Erfüllung von (7) ist wegen $(20)$ und $G^{\left(k, i_{0}\right)} \subset H^{(k)} \subset\left(2^{k} ; 2^{k+1}\right]$

Die Erfüllung der 7) Eigenschaft folgt für $0 \leqslant a \leqslant \frac{1}{4} f(k)$ wegen (21) und $\mathfrak{Z}^{(k)} \subset \mathfrak{Z}^{(k+1)}$ aus der Voraussetzung der Induktion, für $\frac{1}{4} f(k)<$ $\leqslant 2^{k+1}$ aus der Definition von $\mathfrak{U}^{(k+1)} \cap\left(\frac{1}{4} f(k) ; 2^{k+1}\right]$.

Die Lösungzahl von (8) ist der Konstruktion gemäß die Summe der Lösungzahlen (15), (16) und (17) (mit $i=i_{0}$ ), die aber der Konstruktion gemäß kleiner ist, als $\varphi(k+1)$; die 8$)$ Eigenschaft ist also erfüllt.

Wir müssen noch (3) und (4) beweisen; es genügt offensichtlich beides für $i=k+1$ zu beweisen.

Ist $a_{r}^{(k+1)} \epsilon \mathfrak{U}^{(k+1)}, a_{r}^{(k+1)}>\frac{1}{4} f(k)$ und $b_{s}^{(k+1)} \epsilon \mathfrak{Z}^{(k+1)}$, so ist nach der Konstruktion von $\mathfrak{U}^{(k+1)} \cap\left(\frac{1}{4} f(k) ; 2^{k+1}\right] \quad a_{r}^{(k+1)}+b_{s}^{(k+1)} \epsilon \mathfrak{C}$, ferner offensichtlich $a_{r}^{(k+1)}+b_{s}^{(k+1)} \leqslant 2^{k+2}$. Ist $a_{r}^{(k+1)} \in \mathfrak{U}^{(k+1)}, \quad b_{s}^{(k+1)} \epsilon \mathfrak{V}^{(k+1)}, \quad 0 \leqslant a_{r}^{(k+1)}$ $\leqslant \frac{1}{4} f(k)$ und $0 \leqslant b_{s}^{(k+1)} \leqslant 2^{k}$, so ist nach der Konstruktion $a_{r}^{(k+1)} \epsilon \mathfrak{U}^{(k)}$ und $b_{s}^{(k+1)} \epsilon \mathfrak{B}^{(k)}$, wegen der Voraussetzung der Induktion also $a_{r}^{(k+1)}+$ $+b_{s}^{(k+1)} \epsilon \mathfrak{U}^{(k)}+\mathfrak{Z}^{(k)} \subset\left[0 ; 2^{k+1}\right] \frown$ C. Ist schließlich $a_{r}^{(k+1)} \epsilon \mathfrak{U}^{(k+1)}, b_{s}^{(k+1)} \epsilon \mathfrak{Z}^{(k+1)}$ $0 \leqslant a_{r}^{(k+1)} \leqslant \frac{1}{4} f(k)$ und $b_{s}^{(k+1)}>2^{k}$, so ist wegen $b_{s}^{(k+1)} \epsilon G^{\left(k, i_{0}\right)} \subset H^{(k)}$

$$
\left[b_{s}^{(k+1)} ; b_{s}^{(k+1)}+f(k)\right] \frown \mathfrak{C}=\left[b_{s}^{(k+1)} ; b_{s}^{(k+1)}+f(k)\right] \frown \mathfrak{N},
$$

also $a_{r}^{(k+1)}+b_{s}^{(k+1)} \epsilon \mathfrak{C}$, ferner offensichtlich $a_{r}^{(k+1)}+b_{s}^{(k+1)} \leqslant 2^{k+2}$.

Bevor wir den Beweis von (4) liefern, beweisen wir

$$
[0 ; f(k)] \frown \mathfrak{U}^{(k)} \subset[0 ; f(k)] \frown \mathfrak{U}^{(k+1)} .
$$

$\mathrm{Da}(6)$ auch mit $i=k+1$ bewiesen ist, genügt es $\mathrm{zu}$ beweisen, daß für $\frac{1}{4} f(k)<a_{r}^{(k)} \leqslant f(k), \quad a_{r}^{(k)} \epsilon^{\mathfrak{U}^{(k)}}, a_{r}^{(k)} \epsilon \mathfrak{U}^{(k+1)}$ erfüllt ist. Für $0 \leqslant b^{(k+1)}$ $\leqslant 2^{k}, b_{s}^{(k+1)} \in \mathfrak{B}^{(k+1)}$ ist $b_{s}^{(k+1)} \in \mathfrak{Z}^{(k)}$, auf Grund der Voraussetzung der Induktion also

$$
a_{r}^{(k)}+b_{s}^{(k+1)} \epsilon \mathfrak{U}^{(k)}+\mathfrak{B}^{(k)} \subset \mathfrak{C} ;
$$

für $2^{k}<b_{s}^{(k+1)} \leqslant 2^{k+1}, b_{s}^{(k+1)} \in \mathfrak{B}^{(k+1)}$ ist jedoch der Konstruktion gemäß

$$
\left[b_{s}^{(k+1)} ; b_{s}^{(k+1)}+f(k)\right] \frown \mathfrak{C}=\left[b_{s}^{(k+1)} ; b_{s}^{(k+1)}+f(k)\right] \frown \mathfrak{M},
$$

mithin wegen $b_{s}^{(k+1)}<b_{s}^{(k+1)}+a_{r}^{(k)} \leqslant b_{s}^{(k+1)}+f(k) b_{s}^{(k+1)}+a_{r}^{(k)} \epsilon \mathfrak{C}$. So ist für jedes $b_{s}^{(k+1)} \in \mathfrak{B}^{(k+1)} a_{r}^{(k)}+b_{s}^{(k+1)} \in \mathcal{E}$, es gilt also entsprechend der Konstruktion von $\mathfrak{U}^{(k+1)} \frown\left\langle\frac{1}{4} f(k) ; 2^{k+1}\right| a_{r}^{(k)} \epsilon \mathfrak{U}^{(k+1)}$, und damit (22) haben wir bewiesen.

Nun gehen wir zum Beweis von (4) über. Aus der Voraussetzungen der Induktion und (22) folgt

$$
\begin{aligned}
(\mathfrak{S} \cap[0 ; f(k)] & \subset\left(\mathfrak{U}^{(k)} \cap[0 ; f(k)]\right)+\left(\mathfrak{Z}^{(k)} \cap[0 ; f(k)]\right) \\
& \subset\left(\mathfrak{U}^{(k+1)} \frown[0 ; f(k)]\right)+\left(\mathfrak{Z}^{(k+1)} \frown[0 ; f(k)]\right) \\
& \subset \mathfrak{U}^{(k+1)}+\mathfrak{Z}^{(k+1)} .
\end{aligned}
$$

Es sei nun $f(k)<c_{r} \leqslant 2^{k+1}, c_{r} \in \mathfrak{C}$.

Nehmen wir an, daß im Gegensatz zur Behauptung $a_{r} \mathscr{U}^{\left({ }^{(k+1)}\right.}+\mathfrak{Z}^{(k+1)}$ ist. Dann ist für $b_{s}^{(k+1)} \in \mathfrak{Z}^{(k+1)}$ und $0 \leqslant b_{s}^{(k+1)}<\frac{3}{4} f(k), \quad c_{r}-b_{s}^{(k+1)} \notin \mathcal{U}^{(k+1)}$ und $\frac{1}{4} f(k)<c_{r}-b_{s}^{(k+1)} \leqslant 2^{k+1}$; demgemäß existiert entsprechend der Konstruktion von $\left(\frac{1}{4} f(k) ; 2^{k+1}\right] \frown \mathfrak{U}^{(k+1)}$ ein $b_{s^{*}}^{(k+1)} \epsilon \mathfrak{Z}^{(k+1)}$ derart, daß (23)

$$
\left(c_{r}-b_{s}^{(k+1)}\right)+b_{s^{*}}^{(k+1)}=c_{r}-\left(b_{s}^{(k+1)}-b_{s^{*}}^{(k+1)}\right) \notin \mathfrak{C}
$$

erfüllt ist.

Es folgt aus (5) (mit $i=k+1, s=s_{0}$, wobei $2^{s_{0}}<\frac{3}{4} f(k) \leqslant 2^{s_{0}+1}$ ist), daß für hinreichend großes $k_{0}$

$$
B\left(\frac{3}{4} f(k)\right) \geqslant\left[\sqrt{\frac{3}{8} f(k)}\right]>\frac{1}{2} \sqrt{f(k)}
$$

gilt. So $s$ kann in (23) mindestens $\frac{1}{2} \sqrt{f(k)}$ Werte annehmen, wird also $s$ in (23) auf die erwähnte Weise verändert, dann nimmt $c_{r}-\left(b_{s}^{(k+1)}-b_{s^{*}}^{(k+1)}\right)$ der 8. Eigenschaft (mit $i=k+1$ ) zufolge mindestens $\frac{1}{2} \sqrt{f(k)} / \varphi(k+1)$ verschiedene Werte an, die nicht größer sind, als $2^{k+2}$ und in $\mathbb{E}$ nicht enthalten sind, also Werte, die in $\overline{\mathfrak{C}} \cap\left[0 ; 2^{k+2}\right]$ enthalten sind. Für hinreichend großes $k_{0}$ ist aber

$$
\begin{aligned}
\frac{\sqrt{f(k)}}{2 \varphi(k+1)} & =\frac{\sqrt{\frac{1}{12 c} \sqrt[3]{\frac{2^{2 k}\left(\log 2^{k}\right)^{4}}{\left(\log \log 2^{k}\right)^{2}}}}}{2 \cdot \frac{1}{32 c^{3 / 2} \cdot \frac{\left(\log 2^{k+1}\right)^{2}}{\log \log 2^{k+1}}}} \\
& >c \frac{16}{\sqrt{13}} \sqrt[3]{2^{k} \frac{\left(\log \log 2^{k}\right)^{2}}{\left(\log 2^{k}\right)^{4}}}>40 \sqrt[3]{\frac{2^{k}\left(\log \log 2^{k}\right)^{2}}{\left(\log 2^{k}\right)^{4}}}>\bar{C}\left(2^{k+2}\right),
\end{aligned}
$$

womit wir $z u$ einem Widerspruch gelangt sind.

Nunmehr können wir auf die Konstruktion eingehen.

Es ist leicht ersichtlich, daß für $k \geqslant 4$

$$
f(k+1)>f(k) \quad(k \geqslant 4)
$$

ist; es sei $k_{1}=\max \left\{4, k_{0}\right\}$.

Es sei

und

$$
\mathfrak{U} \stackrel{\text { def }}{=} \bigcup_{k=k_{1}}^{+\infty}\left(\mathcal{U}^{(k)} \frown\left[0 ; \frac{1}{4} f(k)\right]\right)
$$

$$
\mathfrak{B} \stackrel{\text { def }}{=} \bigcup_{k=k_{0}}^{+\infty} \mathfrak{Z}^{(k)} \text {. }
$$

Wir müssen beweisen, daß

$$
\mathfrak{U}+\mathfrak{Z}=\mathfrak{C}
$$

ist, und die Folgen $\mathfrak{U}$ und $\mathfrak{B}$ mindestens zwei Elemente enthalten. 
(25) ist auf Grund von (3), (4), (6), (7) und (24) leicht zu beweisen. Aus (5) und (7) folgt

$$
\lim _{n=+\infty} \inf \frac{B(n)}{\sqrt{n}}>0
$$

und

$$
B(n)=O(\sqrt{n}) .
$$

Aus der letzten Behauptung folgt wegen $A(n) B(n) \geqslant C(n)$ und $O(n) \sim n$

$$
\lim _{n=+\infty} \inf \frac{A(n)}{\sqrt{n}}>0 .
$$

Aus (26) und (27) ergibt sich, daß $\mathfrak{U}$ und $\mathfrak{Z}$ unendlich viele Elemente enthalten, und damit ist der Satz bewiesen.

Wir bemerken noch, daß die im Satz vorkommende Konstante o auch explizit berechenbar ist.

Es verlohnt sich, eine triviale Konsequenz des Satzes besonders zu erwähnen:

Satz II. Ist für eine Folge $\mathbb{C}$

$$
\lim _{n=+\infty} \sup (n-C(n)) \sqrt[3]{\frac{(\log n)^{4}}{n(\log \log n)^{2}}}<c
$$

(wobei c die im Satz I. vorkommende Konstante ist), so ist die Folge nicht totalprimitiv.

\section{Literaturverzeichnis}

[1] A. Sárközy, Über totalprimitive Folgen, Acta Arithm. 8 (1962), S. 21-31.

Reşu par la Rédaction le 18. 2. 1964

\section{Some partition problems}

related to the Stirling numbers of the second kind

by

L. Carlitz (Durham, N. C.)*

1. Put ([4], Ch. 4$)$

$$
\exp \left\{t\left(e^{x}-1\right)\right\}=\sum_{n=0}^{\infty} A_{n}(t) \frac{x^{n}}{n !},
$$

so that

$$
A_{n}(t)=\sum_{r=0}^{n} a(n, r) t^{r}
$$

where

$$
a(n, r)=\frac{1}{r !} \sum_{j=0}^{r}(-1)^{r-j}\left(\begin{array}{l}
r \\
j
\end{array}\right) j^{n} .
$$

The $a(n, r)$ are the Stirling numbers of the second kind.

For fixed $n$, let $\theta_{0}(n)$ denote the number of $a(n, 2 r), 0 \leqslant 2 r \leqslant n$, that are odd and put

$$
\theta_{0}(n+2)=\omega_{0}(n)
$$

The writer has proved ([1], [2]) that $\omega_{0}(n)$ satisfies

$$
\sum_{n=0}^{\infty} \omega_{0}(n) x^{n}=\prod_{n=0}^{\infty}\left(1+x^{2^{n}}+x^{2^{n+1}}\right)
$$

and derived a number of additional properties of $\omega_{0}(n)$.

In the present paper we consider the corresponding problems for other prime moduli. Let $\theta_{j}(n)$ denote the number of $a(n, k), 0 \leqslant k \leqslant n$, that are prime to $p$ and such that

$$
k \equiv j(\bmod p) \quad(0 \leqslant j \leqslant p-1),
$$

* Supported in part by National Science Foundation grant GP-1593. 